\title{
Domain 2 of Drosophila Para Voltage-Gated Sodium Channel Confers Insect Properties to a Rat Brain Channel
}

\author{
Iris Shichor, ${ }^{1,3}$ Eliahu Zlotkin, ${ }^{3}$ Nitza Ilan, ${ }^{1,2}$ Dodo Chikashvili, ${ }^{1}$ Walter Stuhmer, ${ }^{4}$ Dalia Gordon, ${ }^{2}$ and \\ Ilana Lotan ${ }^{1}$
}

Departments of ${ }^{1}$ Physiology and Pharmacology, Sackler School of Medicine and 2 Plant Sciences, George S. Wise Faculty of Life Sciences, Tel-Aviv University, 69978 Ramat-Aviv, Israel, ${ }^{3}$ Department of Cell and Animal Biology, Institute of Life

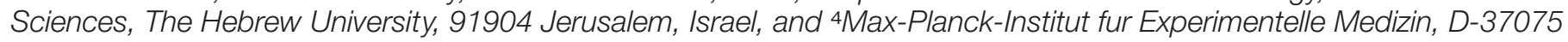
Gottingen, Germany

\begin{abstract}
The ability of the excitatory anti-insect-selective scorpion toxin AahIT (Androctonus australis hector) to exclusively bind to and modify the insect voltage-gated sodium channel ( $\mathrm{NaCh}$ ) makes it a unique tool to unravel the structural differences between mammalian and insect channels, a prerequisite in the design of selective pesticides. To localize the insect $\mathrm{NaCh}$ domain that binds AahlT, we constructed a chimeric channel composed of rat brain $\mathrm{NaCh} \alpha$-subunit (rBIIA) in which domain-2 (D2) was replaced by that of Drosophila Para (paralytic temperaturesensitive). The choice of D2 was dictated by the similarity between AahlT and scorpion $\beta$-toxins pertaining to both their binding and action and the essential role of D2 in the $\beta$-toxins binding site on mammalian channels. Expression of the chimera rBIIA-ParaD2 in Xenopus oocytes gave rise to voltage-gated and TTX-sensitive NaChs that, like rBIIA, were sensitive to
\end{abstract}

scorpion $\alpha$-toxins and regulated by the auxiliary subunit $\beta_{1}$ but not by the insect TipE. Notably, like Drosophila Para/TipE, but unlike $\mathrm{rBII} / \beta_{1}$, the chimera gained sensitivity to AahIT, indicating that the phyletic selectivity of AahIT is conferred by the insect $\mathrm{NaCh}$ D2. Furthermore, the chimera acquired additional insect channel properties; its activation was shifted to more positive potentials, and the effect of $\alpha$-toxins was potentiated. Our results highlight the key role of D2 in the selective recognition of anti-insect excitatory toxins and in the modulation of $\mathrm{NaCh}$ gating. We also provide a methodological approach to the study of ion channels that are difficult to express in model expression systems.

Key words: Na channel; insect selectivity; Xenopus oocytes; scorpion toxin; gating; Drosophila Para
Voltage gated sodium channels ( $\mathrm{NaChs}$ ) play a pivotal role in excitability. They are plasma membrane proteins composed of the large pore-forming $\alpha$-subunit generally accompanied by one or two auxiliary subunits (Catterall, 1992; Feng et al., 1995). The $\alpha$-subunit is composed of four repeated domains (D1-D4) consisting of six transmembrane $\alpha$-helixes (S1-S6) and a pore loop (between S5 and S6) (Catterall, 1995). Many modifiers of NaChs are used as drugs and insecticides (Kallen et al., 1993; Gordon, 1997a,b). However, being unable to distinguish between $\mathrm{NaCh}$ subtypes in various tissues and species, their use often renders adverse side effects or toxicity to nontargeted animals. However, some scorpion neurotoxins are able to exclusively identify insect NaChs (Zlotkin et al., 1978; Zlotkin, 1999) or distinguish among distinct mammalian neuronal subtypes (Gilles et al., 1999, 2000). Such selectivity indicates structural differences at toxin binding sites among $\mathrm{NaChs}$ that, when identified, can be used in the design of selective drugs and insecticides.

\footnotetext{
Received Jan. 17, 2002; revised March 17, 2002; accepted March 12, 2002.

This work was partly supported by Israeli Science Foundation Grants 437/98 (I.L.) and 508/00 (D.G.), United States-Israel Binational Science Foundation Grant 1999396 (I.L.), and German-Israeli Foundation Grant I 0375-172 (W.S., E.Z., I.L., and D.G.). We thank Nathan Dascal for helpful discussions and Jeffrey Warmke and Martin Williamson for the kind gifts of the Drosophila Para and TipE clones, respectively.

Correspondence should be addressed to either Prof. Ilana Lotan, Department of Physiology and Pharmacology, Sackler School of Medicine, Tel-Aviv University, 69978 Ramat-Aviv, Israel, E-mail: ilotan@post.tau.ac.il; or Dr. Dalia Gordon, Department of Plant Sciences, George S. Wise Faculty of Life Sciences, Tel-Aviv University, 69978 Ramat-Aviv, Israel, E-mail: dgordon@post.tau.ac.il.

Copyright (ㄷ) 2002 Society for Neuroscience $0270-6474 / 02 / 224364-08 \$ 15.00 / 0$
}

Scorpion toxins affecting $\mathrm{NaChs}$ bind to two receptor sites on the $\alpha$-subunit and are divided into $\alpha$ - and $\beta$-classes according to their mode of action and binding properties (Gordon et al., 1998). $\alpha$-Toxins inhibit sodium current inactivation during binding to receptor site-3, which involves extracellular loops S5-S6 of D1 and D4 and S3-S4 of D4 (Thomsen and Catterall, 1989; Rogers et al., 1996; Ma et al., 2000). $\beta$-Toxins shift the voltage dependence of activation to more negative potentials (Wang and Strichartz, 1983) (for review, see Gordon, 1997b) by binding to receptor site-4 (Jover et al., 1980), shown to be in domain-2 (D2) (Marcotte et al., 1997; Tsushima et al., 1999) and to include the external loop S3-S4 (Cestele et al., 1998).

Two additional groups of scorpion toxins, the excitatory and depressant, are highly selective to insects (Zlotkin et al., 1978; Zlotkin, 1997). Like the $\beta$-toxins acting on mammals, the excitatory and depressant toxins modify the activation of insect $\mathrm{NaChs}$ and compete for binding with other $\beta$-toxins (for review, see Gordon et al., 1998), properties that suggest their affiliation to the $\beta$-toxin class. The exclusive recognition of insect $\mathrm{NaChs}$ was best demonstrated using the excitatory toxin from Androctonus australis hector (AahIT). AahIT is composed of 70 amino acids cross-linked by four disulfide bridges (Zlotkin et al., 1978) and modifies the gating mechanism by binding to an external receptor site of insect NaChs (Pelhate and Zlotkin, 1981, 1982; Gordon et al., 1984, 1992). Its strict selectivity for insects, which has been documented by toxicity and electrophysiological and binding experiments (Gordon, 1997a; Zlotkin, 1997), makes AahIT a 
unique tool to study the basis of selective recognition of insect channels.

To clarify the basis of the selective interaction of AahIT with insect $\mathrm{NaChs}$, aiming to localize the region that is targeted by AahIT, we constructed a chimeric mammalian-insect channel of the rat brain $\mathrm{NaCh}$ IIA (rBIIA) in which D2 was replaced with that of the Para (paralytic temperature-sensitive) Drosophila channel. The chimeric channel was functional, despite the large phylogenetic distance between insects and mammals, and acquired the sensitivity to the anti-insect selective toxin AahIT.

\section{MATERIALS AND METHODS}

\section{Materials}

Para NaCh DNA was a kind gift from Dr. J. Warmke (Merck, Rahway, NJ). The clone of TipE was a kind gift from Dr. M. Williamson (IACRRothamsted, Harpenden, UK). AahIT was purified by column chromatography from the crude venom of the North African scorpion Androctonus australis hector as described previously (Zlotkin et al., 1971). Lqh $\alpha$ IT, Lqh-II (Ltx001) (from Leiurus quinquestratus hebraeus) was purchased from Latoxan (Valence, France). TTX was purchased from Sigma (Jerusalem, Israel). All reagents used were of molecular biology grade.

\section{Construction of a chimeric cDNA, rBIIA-ParaD2}

The rat brain NaCh $\alpha$-subunit cDNA (rBIIA, pVA2580) (Gershon et al., 1992) was restricted by $X b a \mathrm{I}$ and $B g l \mathrm{II}$ to excise a 1233 bp fragment corresponding to D2 and was isolated by gel electrophoresis. The $1380 \mathrm{bp}$ parallel fragment of Drosophila Para cDNA corresponding to D2 was amplified by PCR using Para Drosophila cDNA as a template and two primers containing either $X b a \mathrm{I}$ or $B g l \mathrm{II}$ restrictions sites corresponding to those of the rat brain cDNA: primer 1 (sense), 5'- GCCTCCCGGGGGTCGTATACCTCACATGGCGATCTACTCGGC- ' 3 ; and primer 2 (antisense), 5'-GGACAGATCTTCCAGTTGCGTCTGCTCCTTGATCCC-'3.

The PCR conditions were as follows: 27 cycles of $96^{\circ} \mathrm{C}$ for $1 \mathrm{~min}, 62^{\circ} \mathrm{C}$ for $45 \mathrm{sec}$, and $72^{\circ} \mathrm{C}$ for $2 \mathrm{~min}$, using the PFU DNA polymerase (Stratagene, La Jolla, CA). The 1380 bp PCR product cDNA was restricted with $X b a \mathrm{I}$ and $B g l \mathrm{II}$, isolated by agarose gel electrophoresis, extracted by gel purification kit (QIAEX; Qiagen, Hilden, Germany), and ligated (T4 DNA ligase; New England Biolabs, Beverly, MA) to the excised rat brain $\mathrm{NaCh}$ cDNA lacking D2. The ligation products were transformed into competent bacteria cells (Escherichia coli; JM-109; Promega, Madison, WI). Colonies were examined by restriction analysis, and relevant ones were sequenced (automated sequencer; core facilities at Tel-Aviv University).

\section{Generation of $c R N A$, injection into Xenopus oocytes, and electrophysiology}

rBIIA cRNA was generated from the pVA2580 construct, linearized by ClaI, and transcribed in vitro with T7 RNA polymerase as described previously (Dascal and Lotan, 1992; Gershon et al., 1992). Chimeric cRNA $\alpha$-subunit was generated from the rBIIA-ParaD2 cDNA, linearized by $P a c I$, and transcribed with T7 RNA polymerase. $\beta_{1}$-Subunit cRNA was linearized by NotI and transcribed in vitro with T7 RNA polymerase (Wallner et al., 1993).

Para and TipE cRNAs were generated from the pGH19-13-5 Para (Warmke et al., 1997) and pGHTipE (Vais et al., 2000) constructs, respectively, linearized by NotI, and transcribed with T7 RNA polymerase. Oocytes were injected with $0.3-0.8 \mathrm{ng}$ of rBIIA cRNA or with 4-10 ng of rBIIA-ParaD2 cRNA with or without $\beta_{1}$ cRNA (1:1 ratio) (Catterall, 1995; Makita et al., 1996) or with 10 ng of Para cRNA with TipE (1:1 ratio) (Warmke et al., 1997; Vais et al., 2000). Injected oocytes were incubated at $22^{\circ} \mathrm{C}$ for 3-4 d in ND96 solution (in mM: $96 \mathrm{NaCl}, 2 \mathrm{KCl}$, $1 \mathrm{MgCl}_{2}$, and 5 HEPES, pH 7.5) supplemented with $1.8 \mathrm{mM} \mathrm{CaCl}_{2}, 2.5$ $\mathrm{mm}$ sodium pyruvate, and $100 \mu \mathrm{g} / \mathrm{ml}$ gentamicin, as described previously (Gilles et al., 1999).

Sodium currents were recorded using a Warner Instruments (Hamden, CT) OC-725B two-electrode voltage-clamp amplifier with a series resistance compensation circuit and low-resistance agarose-plugged electrodes filled with $3 \mathrm{M} \mathrm{KCl}(0.2-0.5 \mathrm{M} \Omega)$. Experiments were done in a 150 $\mu \mathrm{l}$ bath in ND96 solution supplemented with $1 \mathrm{~mm} \mathrm{CaCl2,} \mathrm{pH} 7.6$, at $20-22^{\circ} \mathrm{C}$. Sodium currents were measured in single oocytes before and after application of the relevant toxin. Toxins were kept in concentrated stock solutions and were diluted before the experiment in ND96 solution that contained $1 \mathrm{mg} / \mathrm{ml} \mathrm{BSA}$. The application of the toxin-BSA solution was done directly into the bath, in a volume up to $15 \mu \mathrm{l}$ (10\% of the bath volume) to get the desired total concentration. To discard any application artifacts, in few oocytes, $1 \mathrm{mg} / \mathrm{ml}$ BSA solution was applied before the application of the toxin. Before impaling the oocyte with electrodes, the voltage base line was set to $0 \mathrm{mV}$. At the end of each experiment, the base line voltage was checked not to exceed $\pm 2 \mathrm{mV}$, otherwise the experiment was discarded. The currents were filtered at $4 \mathrm{kHz}$ and sampled at 11 $\mathrm{kHz}$. Stimulation and data acquisition were done with an IBM computer using the pClamp software (Axon Instruments, Foster City, CA). Net current was estimated by subtraction of scaled leak current.

\section{Data analysis}

Voltage dependence of activation and inactivation. Current-voltage relationship $(I-V)$ data and its transforming to activation curve, i.e., $G / G_{\max }$ versus $V_{\text {test }}$, were fitted using a nonlinear least-square algorithm, to the modified Bolzmann equation (as by Dascal and Lotan, 1991) as follows: $G / G_{\max }=1 /\left\{1+\exp \left[-\left(V_{\text {test }}-V_{1 / 2}\right) / k\right]\right\}^{3}$ (Eq. 1), where at each membrane voltage $\left(V_{\text {test }}\right)\left[G \stackrel{\text { mest }}{=} I /\left(V_{\text {test }}-V_{\text {rev }}\right)\right], I$ is the peak sodium current, $V_{\text {test }}$ is the test potential, and $V_{\text {rev }}$ is the reversal potential. The three free parameters were $V_{1 / 2}$ (the voltage at which the probability of a single gate opening is one-half), $k$ (the slope factor, which corresponds to a change in voltage that produces an $e$-fold change in conductance), and $G_{\max }$ (the maximal $\mathrm{Na}^{+}$conductance attained at very positive voltages). Steady-state inactivation was studied by holding the oocyte at $-80 \mathrm{mV}$ and stepping the voltage for $200 \mathrm{msec}$ to various values from $-90 \mathrm{mV}$ up to $-10 \mathrm{mV}$ in $10 \mathrm{mV}$ increments ( $V_{\text {prepulse }}$ ), before measuring $I_{\mathrm{Na}}+$ by a $50 \mathrm{msec}$ voltage step that elicits maximal current $(-10 \mathrm{mV}$ for the wild-type rBIIA or $0 \mathrm{mV}$ for the rBIIA-ParaD2). Fractional current $\left(I / I_{\max }\right)$ was plotted as a function of $V_{\text {prepulse }}$ and fitted (as by Dascal and Lotan, 1991) to the following equation: $I / I_{\max }=1 /\left\{1+\exp \left[\left(V_{\text {prepulse }}-\right.\right.\right.$ $\left.\left.\left.V_{1 / 2}\right) / k\right]\right\}$ (Eq. 2). $I_{\max }$ was the current obtained by a prepulse to $-90 \mathrm{mV}$, and all other parameters was as above.

Analysis of the effect of $\alpha$-toxins. Two parameters were checked to analyze the effect of the $\alpha$-toxins. The first was the time constant of inactivation of the current described by the sum of two exponents (a fast component $\tau_{1}$ and a slow component $\tau_{2}$ ) and calculated in the same oocyte before and after application of the toxin. The second parameter was the estimated affinity $\left(\mathrm{k}_{\mathrm{D}}\right)$ of the toxin to the channel, determined by the fraction of the maximal peak current (elicited at -10 or $0 \mathrm{mV}$ for rBIIA or rBIIA-ParaD2, respectively) remaining $5 \mathrm{msec}$ after the peak, before, and $13 \mathrm{~min}$ after application of the toxin. Because the fraction of conductance remaining $5 \mathrm{msec}$ after the peak is proportional to the number of channels modified by $\alpha$-toxins (Rogers et al., 1996), it can be used to estimate receptor occupancy and toxin affinity according to the following formula: $K_{\mathrm{D}}=$ [ $\alpha$ toxin $]\left(F_{\mathrm{G}}^{\prime} / F_{\mathrm{G}}-1\right)(\mathrm{Eq} .3)$, where $F_{\mathrm{G}}$ is the fraction of $\mathrm{Na}^{+}$current remaining $5 \mathrm{msec}$ after beginning of the pulse, and $F_{\mathrm{G}^{\prime}}$ is the maximum fraction of current $5 \mathrm{msec}$ after the beginning of the pulse in the presence of a saturating concentration of $\alpha$-toxin.

\section{RESULTS \\ rBIIA is not sensitive to the excitatory insect-selective toxin AahlT in contrast to Drosophila Para}

The rat brain rBIIA (Noda et al., 1986; Auld et al., 1988, 1990) and the insect Drosophila Para NaChs (Loughney et al., 1989; Warmke et al., 1997) have been functionally expressed in Xenopus oocytes, and their biophysical properties were well characterized. We examined the sensitivity of these two channels, expressed in Xenopus oocytes, to the scorpion anti-insect excitatory toxin AahIT, which is toxic to Drosophila flies $\left(\mathrm{PD}_{50}\right.$ of 2.3 $\mathrm{ng} / \mathrm{mg}$ ) (Zlotkin et al., 1999). AahIT (3-10 $\mu \mathrm{M})$ had no effect on $\mathrm{Na}^{+}$current mediated by rBIIA, whereas 1-2 $\mu \mathrm{M}$ AahIT increased significantly the $\mathrm{Na}^{+}$current mediated by the Drosophila Para (Fig. 1) (for detailed characterization, see Fig. 4C). We aimed to confer AahIT sensitivity to the mammalian rBIIA by insertion of an insect channel domain from the Drosophila Para. AahIT shares biophysical and binding properties with other scorpion toxins of the $\beta$-class (Gordon et al., 1984, 1998; Zlotkin et 


\section{Para $\quad$ rBllA}

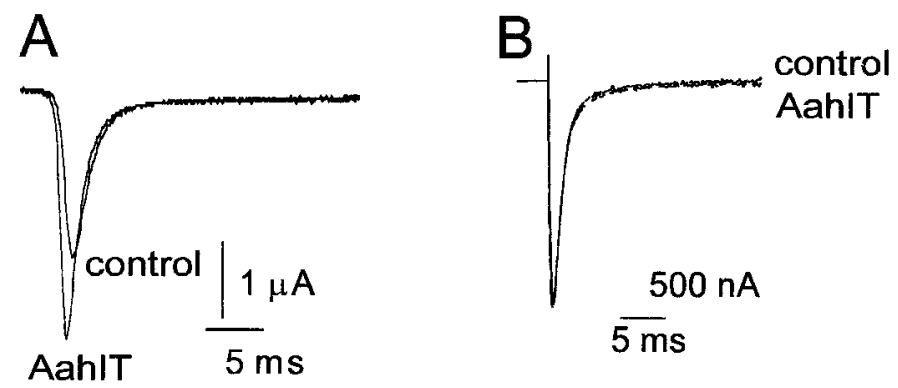

Figure 1. rBIIA is not sensitive to the excitatory insect selective toxin AahIT, in contrast to Drosophila Para. Oocytes expressing the Drosophila Para channel (together with the TipE subunit; $A$ ) or the rBIIA channel (together with the $\beta_{1}$-subunit; $B$ ) were voltage clamped at a holding potential of $-80 \mathrm{mV}$, and currents were elicited by a depolarizing pulse to $-10 \mathrm{mV} . \mathrm{Na}^{+}$currents in single oocytes were recorded before (control) and 13 min after application of $1 \mu \mathrm{M}(A)$ or $3 \mu \mathrm{M}(B)$ AahIT.

al., 1985; De Lima et al., 1986; Lazdunski et al., 1986; Lee and Adams, 2000) (see Discussion). Because the receptor binding site for $\beta$-toxins (site-4) was localized to D2 on mammalian $\mathrm{NaChs}$ (Marcotte et al., 1997; Cestele et al., 1998), we constructed a chimeric channel rBIIA-ParaD2 formed from rBIIA (as a mammalian acceptor channel) and D2 of Para (as a donor of insect channel; see Materials and Methods).

\section{Biophysical characterization of the chimeric channel rBIIA-ParaD2}

Expression of the chimeric $\alpha$-subunit rBIIA-ParaD2 in Xenopus oocytes gave rise to voltage-gated and TTX-sensitive $\mathrm{Na}^{+}$currents (Fig. $2 A, B$ ), indicating that $\mathrm{NaCh}$ activity was not disrupted by the insertion of the insect D2. Like rBIIA (Kontis and Goldin, 1993), the chimeric currents were modulated by coexpression of the mammalian auxiliary $\beta_{1}$-subunit; namely, the inactivation was faster and the amplitudes increased (Isom et al., 1994; Makita et al., 1996) (Fig. $2 A, B$ ). This result indicates that the interaction between the mammalian $\alpha$ - and $\beta 1$-subunits was not interrupted by the insect D2, in concert with the documented involvement of D4 in this interaction (Qu et al., 1999). Notably, coexpression of the insect auxiliary subunit TipE, required for the functional expression of the Drosophila Para $\mathrm{NaCh} \alpha$-subunit in Xenopus oocytes (Feng et al., 1995; Warmke et al., 1997), had no effect on the chimeric channel activity (data not shown). Thus, all subsequent electrophysiological experiments were performed with coexpressed $\alpha$ - and $\beta_{1}$-subunits. To obtain the same amplitudes of $\mathrm{Na}^{+}$currents, 10-fold higher cRNA concentration of rBIIAParaD2 was required compared with that of rBIIA (see Materials and Methods). Expression of both proteins in the reticulocyte lysate system (Jing et al., 1999) revealed that rBIIA protein level of expression was $\sim 10$-fold higher than that of rBIIA-ParaD2 (data not shown), suggesting that, at least partly, impaired expression of rBIIA-ParaD2 is responsible for the low current amplitudes.

Comparison between rBIIA and rBIIA-ParaD2 biophysical characteristics revealed a positive shift of $+11 \mathrm{mV}$ in the halfactivation voltage $\left(V_{1 / 2}\right)$ of the chimera, accompanied by an increase of $2.8 \mathrm{mV}$ in the slope factor $(k)$, compared with rBIIA (Fig. 2C,D), and both effects were statistically highly significant $(p<0.001)$. The activation characteristics of the rBIIA-ParaD2 $\left(V_{1 / 2}=-13 \mathrm{mV} ; k=5.1 \mathrm{mV}\right)($ Fig. $2 D)$ were similar to those of the Para Drosophila $\mathrm{Na}^{+}$channel, which was previously fully characterized in oocytes (Warmke et al., 1997). The $\alpha$-subunit of the Para Drosophila, expressed with TipE, was shown to have $V_{1 / 2}$ $=-16.9 \mathrm{mV}$ and $k=5.43 \mathrm{mV}$. Thus, it seems that the rBIIAParaD2 chimera acquired insect channel properties related to voltage-dependent activation, which is in concert with the importance of D2 in the voltage sensitivity of the NaChs (Auld et al., 1990; Marcotte et al., 1997; Qu et al., 1999). The steady-state inactivation characteristics of the rBIIA-ParaD2 were slightly different, but statistically significant, from those of rBIIA (Fig. $2 E$ ) and were within the range of values determined for the Drosophila Para channel expressed in Xenopus oocytes (Warmke et al., 1997).

\section{rBIIA-ParaD2 is sensitive to AahIT}

Comparison of the effects of AahIT on rBIIA and rBIIA-ParaD2 channels (Fig. 3) revealed that $3 \mu \mathrm{M}$ AahIT had no effect on rBIIA currents at all test potentials, as shown in the $I-V$ relationship (Fig. 3A, bottom). In contrast, $1.4 \mu \mathrm{M}$ AahIT increased markedly the rBIIA-ParaD2 current, as shown for currents elicited at -30 and $-20 \mathrm{mV}$ (Fig. $3 B$, top). Analysis of the voltage dependence of activation of the rBIIA-ParaD2 revealed a statistically significant $\left(p \leq 0.003\right.$ ) negative shift of $6 \mathrm{mV}$ in $V_{1 / 2}$ in the presence of AahIT (Fig. 3B, middle and bottom). This effect resembles the negative shift in the voltage dependence of activation of $\mathrm{Na}^{+}$currents in insect neurons induced by AahIT (Lee and Adams, 2000). As described previously (Marcotte et al., 1997; Cestele et al., 1998; Tsushima et al., 1999), such an effect is typical to $\beta$-toxins acting on mammalian $\mathrm{Na}^{+}$channels.

\section{Conditioning pulse potentiates the effect of AahlT on the rBIIA-ParaD2 channel}

Depolarizing conditioning pulse was demonstrated to be crucial for the activity of the $\beta$-toxin Css-IV (from Centruroides suffusus suffusus) on the rat brain NaCh rBIIA (Cestele et al., 1998) or to potentiate the effect of other $\beta$-toxins on mammalian $\mathrm{NaChs}$ (Tsushima et al., 1999). Based on the results showing that AahIT belongs to the $\beta$-toxin class (Fig. 3), we wanted to examine the effect of depolarizing conditioning pulse on the activity of AahIT. To this end, we compared in single oocytes the effect of AahIT with and without a $2 \mathrm{msec}$ depolarizing prepulse to $+50 \mathrm{mV}$ (Cestele et al., 1998). The results obtained in a representative oocyte clearly show that the prepulse potentiated the effect of AahIT on rBIIA-ParaD2 currents (Fig. 4A, compare - prepulse with + prepulse). With the prepulse, AahIT not only shifted the voltage dependence of activation but also increased the maximal current $\left(I_{\max }\right)$. Steady-state activation analysis in several oocytes (Fig. 4B) not only revealed a prepulse-independent shift in the equilibrium activation potential $(\sim 5.5 \mathrm{mV})$ but also an increase in the maximal conductance $\left(G_{\max }\right)$ with the prepulse by $23 \pm 6 \%$ $(n=4 ; p \leq 0.033)$. Thus, using the prepulse, we further revealed the similarity between the effects of AahIT on rBIIA-ParaD2 and that of $\beta$-toxins on mammalian NaChs.

Analysis of the effect of 1-2 $\mu \mathrm{M}$ AahIT on the Drosophila Para (the donor of D2) coexpressed with TipE revealed an increase in the maximal conductance by $35 \pm 10 \%(n=4 ; p \leq 0.019)$ and a shift in the equilibrium activation potential of $\sim 3 \mathrm{mV}$, the former effect being larger and the latter effect being smaller than those observed in the chimera (Fig. $4 C$ ). Notably, the rBIIA channel remained insensitive to AahIT also with the prepulse (Fig. 4D). 
A $\quad \mathrm{BBI} A$

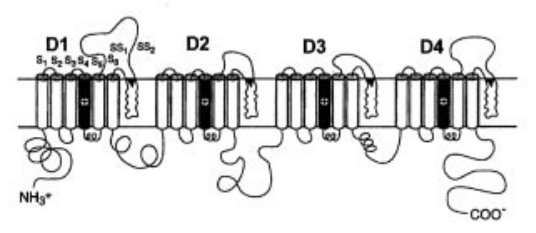

$\alpha$

$$
\alpha+\beta 1
$$
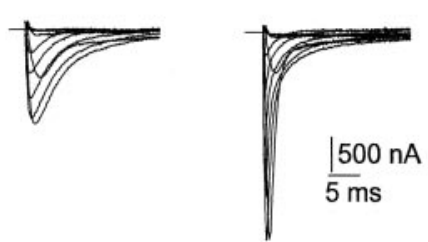

C

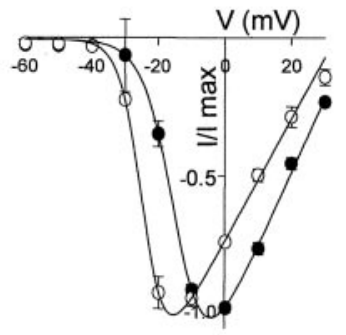

OBIIA

- rBIIA-ParaD2
B

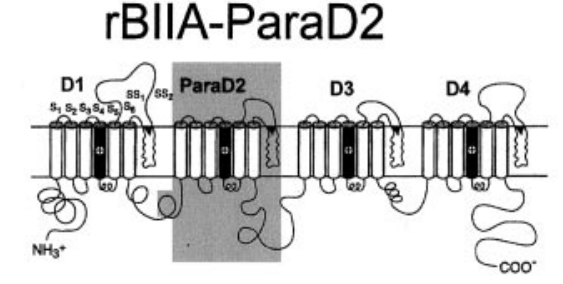

$\alpha$
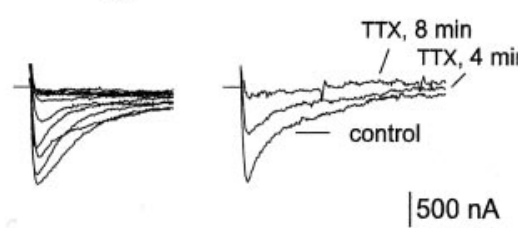

$5 \mathrm{~ms}$

$\alpha+\beta 1$
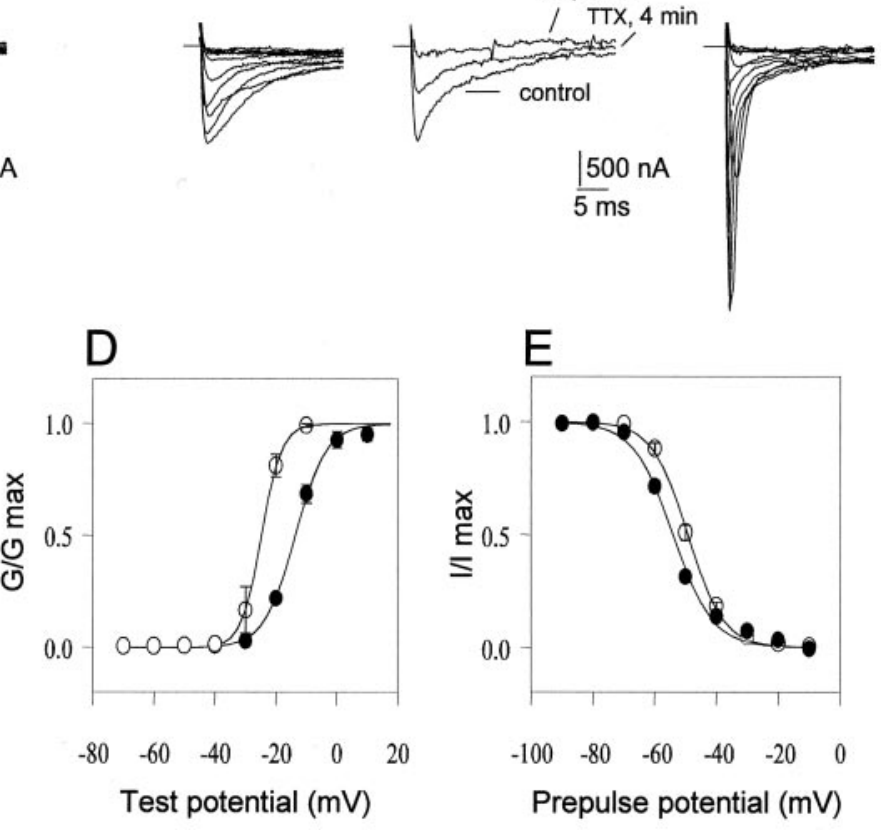

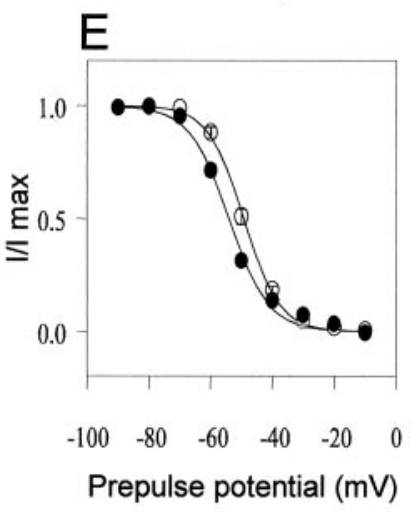

Figure 2. Comparison between the chimera, rBIIA-ParaD2, and the rBIIA currents. $A, B$, Top, A schematic presentation of rBIIA $(A)$ and rBIIA-ParaD2 $(B)$ channel domains (D1-D4), the latter having rBIIA D1, D3, and D4 and the Para Drosophila D2 (ParaD2; gray box). Bottom, $\mathrm{Na}^{+}$currents elicited in oocytes expressing $\alpha$-subunits of rBIIA $(A)$ or rBIIA-ParaD2 $(B)$ alone $(\alpha)$ or together with $\beta_{1}$ - subunit $\left(\alpha+\beta_{1} ; 1: 1\right)$ as denoted. Oocytes were held at -100 $\mathrm{mV}$, followed by $40 \mathrm{msec}$ depolarizations to voltages varying from -80 to $+30 \mathrm{mV}$ in $10 \mathrm{mV}$ increments. Middle trace in $B$ shows rBIIA-ParaD2 currents elicited by stepping to $0 \mathrm{mV}$ from -80 $\mathrm{mV}$ holding potential, before and 4 or 8 min after $1 \mu \mathrm{M}$ TTX application. $C$, Normalized peak $\mathrm{Na}^{+}$currents $\left(I / I_{\max }\right)$ of rBIIA (open circles) and rBIIAParaD2 ( filled circles) plotted as a function of membrane voltage $(V)$. Each value is mean \pm SEM from seven oocytes. The experimental results were fitted using a modified Bolzmann equation (Eq. 1 in Materials and Methods). $D$, Normalized conductance $\left(G / G_{\max }\right)$ voltage relationships derived from the data in $C$. Data were fitted using Equation 1 (see Materials and Methods). Symbols are as in $C$. Values for halfactivation potential $\left(V_{1 / 2}\right)$ and for the slope factor $(k)$ were as follows: rBIIA, $V_{1 / 2}=-24.7 \pm 1.4 \mathrm{mV}, k=2.3 \pm 0.24$ $\mathrm{mV}$; rBIIA-ParaD2, $V_{1 / 2}=-13.6 \pm 0.8$ $\mathrm{mV}, k=5.1 \pm 0.56 \mathrm{mV}$; showing a significant difference between rBIIA and rBIIA-ParaD2 (paired two-tailed $t$ test; $p<0.001) . E$, Steady-state inacti-

vation of the rBIIA (open circles) and rBIIA-ParaD2 ( filled circles). Oocytes were held at $-80 \mathrm{mV}$, and 200 msec steps to prepulse potentials ( $V_{\text {prepulse}}$ ) from -90 to $-10 \mathrm{mV}$ in $10 \mathrm{mV}$ increments were given before eliciting currents by $50 \mathrm{msec}$ steps to -10 or $0 \mathrm{mV}$ (as detailed in Materials and Methods). Fractional current $\left(I / I_{\max }\right)$ was plotted as a function of $V_{\text {prepulse }}$ and fitted to Equation 2 (see Materials and Methods). Each point represents mean \pm SEM values from four oocytes. Values for half-inactivation potential $\left(V_{1 / 2}\right)$ and for the slope factor $(k)$ were as follows: $\mathrm{rBIIA}, V_{1 / 2}=-49 \pm 0.75 \mathrm{mV}$, $k=5.7 \pm 0.12 \mathrm{mV}$; rBIIA-ParaD2, $V_{1 / 2}=-54 \pm 0.7 \mathrm{mV}, k=6.4 \pm 0.13 \mathrm{mV}$; showing a significant difference between rBIIA and rBIIA-ParaD2 (using paired two-tailed $t$ test; $p<0.005$ for $V_{1 / 2} ; p<0.01$ for $k$ ).

\section{Effects of scorpion $\alpha$-toxins on the rBIIA-ParaD2 channel}

Because rBIIA-ParaD2 was found to be sensitive to the insectselective toxin AahIT, we wanted to examine whether this insectmammalian chimera also acquired sensitivity to the $\alpha$-toxin Lqh $\alpha$ IT, which is highly active on insects (Eitan et al., 1990). rBIIA-ParaD2 was poorly sensitive to Lqh $\alpha$ IT; the small decrease in $\tau 2$ (Fig. $5 A$, Table 1 ), however, may be attributed to modifications of intrinsic gating properties rather than to a change in toxin sensitivity caused by the insertion of the insect D2 (Fig. 2) (see below). The rat brain channel rBIIA was practically insensitive to $\mathrm{Lqh} \alpha \mathrm{IT}$, even at concentrations as high as $5 \mu \mathrm{M}$ (Fig. 5A, Table 1), as expected (Gilles et al., 1999). To rule out the possibility that the chimera became resistant to $\alpha$-toxins in general, we tested the effect of the anti-mammalian $\alpha$-toxin Lqh-II (Sautiere et al., 1998; Gilles et al., 1999, 2000). Lqh-II caused a typical inhibitory effect on the inactivation of both rBIIA and rBIIA-ParaD2 currents (Fig. 5B) (Rogers et al., 1996; Gilles et al., 1999, 2001; Chen et al., 2000). The $K_{\mathrm{D}}$ values (a measure of the apparent affinity of the toxin for its receptor) of Lqh-II to the two channels were estimated by measuring the fraction of the current remaining $5 \mathrm{msec}$ after the beginning of the pulse in the absence and presence of the toxin (see Materials and Methods). The estimated $K_{\mathrm{D}}$ values for both channels $(12.78 \pm 4.2 \mathrm{nM}, n=5$; $6.26 \pm 1.76 \mathrm{nM}, n=5$; for rBIIA and rBIIA-ParaD2, respectively) were found not to differ statistically $(p>0.05)$, suggesting that the change in D2 had no major effect on the binding site of the $\alpha$-toxin Lqh-II. Analysis of the effect of Lqh-II on the time constants of inactivation showed a significant increase in the fast $(\tau 1)$ and slow $(\tau 2)$ constants of both rBIIA and rBIIA-ParaD2 channels (Table 1). Notably, the slowing of channel inactivation by Lqh-II was more profound in the rBIIA-ParaD2 channel chimera compared with rBIIA (Table 1, Fig. 5). Moreover, the potentiated effect of Lqh-II on rBIIA-ParaD2 was also manifested by the larger increase in current amplitude [by $3.6 \pm 0.6$ (mean \pm SEM) and $1.76 \pm 0.09$, for rBIIA-ParaD2 and rBIIA, respectively] (Fig. 5B). These results resemble those obtained with another site-3 toxin from sea anemone (ATXII) on Drosophila Para $\mathrm{NaCh}$ expressed in oocytes (Warmke et al., 1997). Despite having comparable affinities to rBIIA and to Drosophila Para, ATXII had a larger effect on the insect channel, manifested in both larger increase in maximal conductance and larger slowing of inactivation. We also verified that $20 \mathrm{~nm}$ Lqh $\alpha$ IT practically abolished inactivation and caused a marked increase in the maximal conductance (data not shown), similar to the effect of ATXII on this insect channel. Together, the high sensitivity of 
A rBIIA
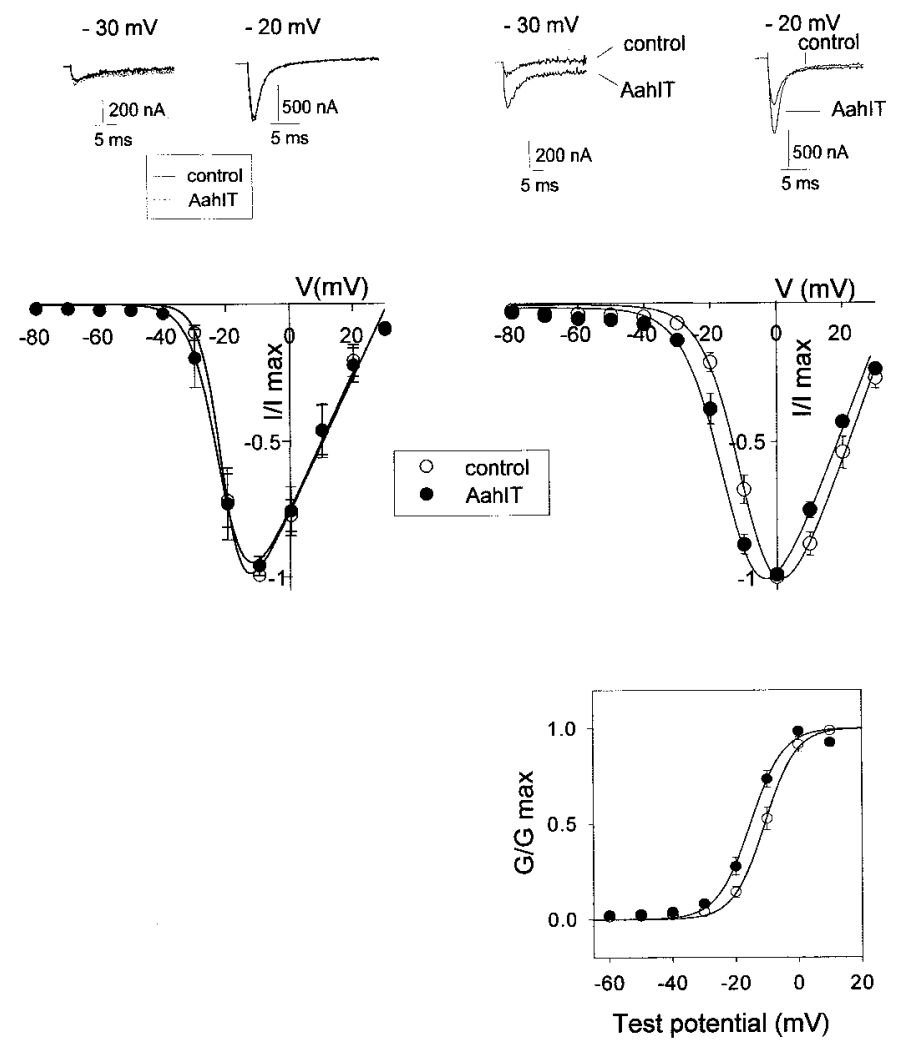

Figure 3. AahIT affects the rBIIA-ParaD2 but not the rBIIA channels. $A, B, T o p$, rBIIA $(A)$ and rBIIA-ParaD2 $(B)$ currents elicited after step depolarizations to -30 or $-20 \mathrm{mV}$ (as denoted above traces) before and 10 min after application of $3 \mu \mathrm{M}(A)$ or $1.4 \mu \mathrm{M}(B)$ AahIT. Concentrations up to $10 \mu \mathrm{M}$ were checked to give the same results. Middle, Normalized peak currents $\left(I / I_{\max }\right)$ of each oocyte plotted as a function of membrane voltage $(V)$, before (open circles, control) and $10 \mathrm{~min}$ after ( filled circles) application of AahIT. Each point represents the mean \pm SEM values from three oocytes treated with $3 \mu \mathrm{M}(A)$ or of six oocytes treated with 1.4-2.8 $\mu \mathrm{M}(B)$ AahIT. Data were fitted using Equation 1 (see Materials and Methods). No significant difference between maximal currents of control and AahIT-treated oocytes was observed. Bottom, Normalized conductance $\left(G / G_{\max }\right)$-voltage relationships derived from the data in the middle panel, fitted to Equation 1. Values for $V_{1 / 2}$ and $k$ were as follows: control, $V_{1 / 2}=-10 \pm 1.2 \mathrm{mV}, k=4.7 \pm 0.15 \mathrm{mV}$; AahIT-treated oocytes, $V_{1 / 2}=-15.5 \pm 1 \mathrm{mV}, k=4.99 \pm 0.07 \mathrm{mV}$; showing a significant difference ( $p \leq 0.003$ using two-tailed paired $t$ test) in $V_{1 / 2}$ between the control and AahIT.

the Para channel, as opposed to the resistance of the rBIIAParaD2, to Lqh $\alpha$ IT and the strong effect of Lqh-II on both rBIIA and the chimera indicate that D2 neither contribute to the binding of receptor site-3 or to the selectivity of $\alpha$-toxins. On the other hand, the larger effects of Lqh-II on the chimeric channel indicate that D2 conferred insect properties related to both activation and inactivation gating.

\section{DISCUSSION}

In this study, using the insect-selective neurotoxin AahIT, we provide a first step toward deciphering the structural elements that are responsible for the selective recognition of insect versus mammalian $\mathrm{NaChs}$ by drugs and toxins. With the background of the close similarity in the primary structure, topological organization, and basic biochemical and pharmacological properties between the mammalian and the insect NaChs (Gordon, 1997a), we used a mammalian-insect chimeric channel to identify the region that is recognized by AahIT and is responsible for its selective insect toxicity.

\section{The choice of the chimera}

The Drosophila Para $\mathrm{NaCh}$, which is sensitive to AahIT (Figs. $1 A$, $4 C$ ), was chosen as a donor of an insect channel region that will confer AahIT sensitivity to the chimeric channel. The rat brain $\mathrm{NaCh}$ rBIIA, shown to be insensitive to high doses of AahIT (Fig. $1 B$ ), was chosen as the mammalian background. Both $\mathrm{NaChs}$ have been well characterized in Xenopus oocytes. To choose the appropriate region of the insect channel, which would confer the sensitivity to AahIT, we considered the following line of evidence that led us to assign AahIT to the $\beta$-toxin class, the receptor site of which is comprised mainly of a single channel domain, D2 (see introductory remarks): (1) like scorpion $\beta$-toxins active on mammals (Cahalan, 1975; Jaimovich et al., 1982; Wang and Strichartz, 1983; Vijverberg et al., 1984; Jonas et al., 1986), AahIT modified the activation process of sodium currents, as shown in cockroach axon (Zlotkin et al., 1985) and in lepidopterous (Heliothis virescens) neurons (Lee and Adams, 2000); (2) AahIT competed with the binding of the $\beta$-toxin Ts-VII (or $\gamma$-toxin, from Tityus serrulatus) (Lazdunski et al., 1986) on cockroach neuronal membranes (De Lima et al., 1986); and (3) like $\beta$-toxins binding to mammalian NaChs (Jover et al., 1980; Lazdunski et al., 1986), AahIT binding to insect (Locusta migratoria) neuronal membranes was not modified by membrane potential or by the alkaloid toxin veratridine (Gordon et al., 1984). Having assigned AahIT to the $\beta$-class of neurotoxins, we assumed that, like on mammalian channels (Marcotte et al., 1997; Cestele et al., 1998; Tsushima et al., 1999), the $\beta$-toxin site on insect channels also resides in D2 and thereby is targeted by AahIT. This assumed that homology between toxin receptor sites on insect and mammalian $\mathrm{NaChs}$ was supported by the corresponding homology demonstrated for the $\alpha$-toxin receptor sites (Gordon and Zlotkin, 1993; Gordon et al., 1996, 2002; Gilles et al., 1999). Thus, we constructed the chimeric channel rBIIA-ParaD2 consisting of rBIIA and D2 of the insect Para Drosophila.

\section{Functional NaCh integrity of the rBIIA-ParaD2 chimera}

rBIIA-ParaD2 formed a functional, voltage-dependent and TTXsensitive $\mathrm{NaCh}$ (Fig. $2 B$ ), despite the large phylogenetic distance between flies and rodents. rBIIA-ParaD2 was also regulated by the coexpressed $\beta_{1}$-subunit (Fig. $2 A, B$ ), consistent with the finding that the binding site for $\beta_{1}$ was localized to D4 in rBIIA (Qu et al., 1999). The chimeric channel was, however, not sensitive to the coexpressed insect auxiliary subunit TipE, excluding the involvement of D2 in the interaction of the insect channel $\alpha$-subunit with TipE. Scorpion $\alpha$-toxin Lqh-II was used to further assess the functional $\mathrm{NaCh}$ integrity of the chimeric channel. The binding of $\alpha$-toxins to an extracellular receptor site (see introductory remarks) affects fast inactivation, a process that involves internal channel regions (Stuhmer et al., 1989; Patton et al., 1992); thus, the toxin effect may reflect a chain of intermolecular changes (Catterall, 1992; Gordon, 1997b; Gilles et al., 2001). The fact that Lqh-II affected the inactivation of the chimeric channel (Fig. 5B) ensured that the overall assembly and activity of the channel were not impaired. This functional $\mathrm{NaCh}$ integrity provided a suitable setting for the study of insect characteristics, including AahIT sensitivity, that were gained by the chimera. 
A



- prepuise
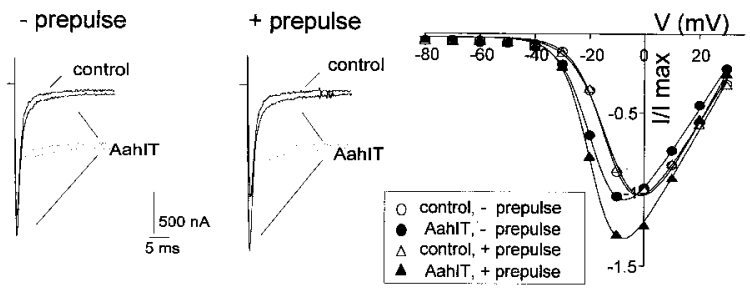

B rBIIA-ParaD2
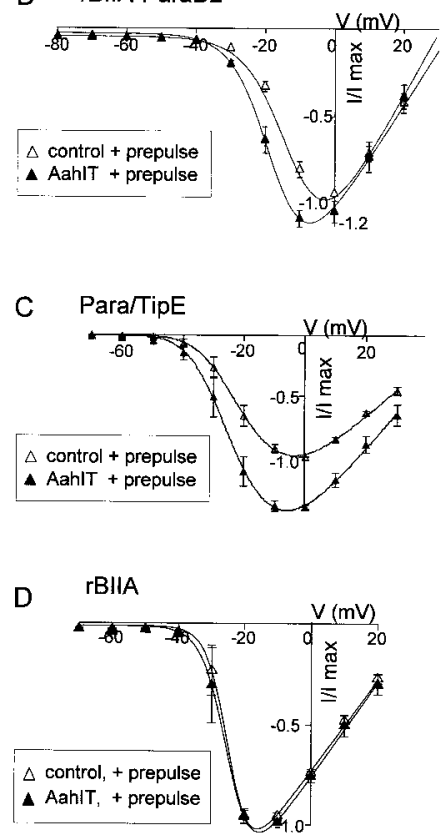

Figure 4. The effect of AahIT is potentiated by a conditioning depolarizing pulse. $A$, rBIIA-ParaD2 currents elicited by step depolarization to $-10 \mathrm{mV}$ without (- prepulse, left) or after (+ prepulse, middle) a $2 \mathrm{msec}$ prepulse to $+50 \mathrm{mV}$, before (control) and after (AahIT) application of 1.4 $\mu \mathrm{M}$ AahIT. Right, Normalized rBIIA-ParaD2 current-voltage relationships measured in a single oocyte, before (open triangles, control) and after (filled triangles) application of $1.4 \mu \mathrm{M}$ AahIT using the prepulse protocol. Data showing the effect of the toxin in the same oocyte without using the prepulse protocol (as in Fig. $3 B$ ) was superimposed (open and filled circles for control and AahIT, respectively). Currents were normalized to maximal current of control. Data were fitted using Equation 1 (see Materials and Methods). $G_{\max }$ values without prepulse were as follows: 53.2 and $52.4 \mu \mathrm{S}$ for control and AahIT-treated oocytes, respectively. $G_{\max }$ values with prepulse were as follows: 43.5 and $50.6 \mu \mathrm{S}$ for control and AahIT-treated oocytes, respectively. B, C, Left, Normalized peak currents $\left(I / I_{\max }\right.$ of control) of rBIIA-ParaD2 $(B)$ or Para/TipE $(C)$ plotted as a function of membrane voltage $(V)$, before (open triangles) and $10 \mathrm{~min}$ after (filled triangles) application of 1-2 $\mu \mathrm{M}$ AahIT, using a prepulse protocol. Each point represents the mean \pm SEM values from four oocytes. Right, Voltage dependence of activation derived from the current-voltage relationships (left) presented as an activation curve of control (open triangles) and AahIT-treated (filled triangles) oocytes. Normalized $G-V$ relationships were fitted using Equation 1 (see Materials and Methods). Values for rBIIA-ParaD2 $(B)$ were as follows: control, $V_{1 / 2}=-14 \pm 0.67 \mathrm{mV}, k=4.7 \pm 0.09 \mathrm{mV}, G_{\max }=31.48 \pm 3$; AahIT-treated oocytes, $V_{1 / 2}=-19.5 \pm 1 \mathrm{mV}, k=4.6 \pm 0.1 \mathrm{mV}, G_{\max }=$ $38.6 \pm 2.6$; showing significant differences (paired two-tailed $t$ test) in $V_{1 / 2}$ $(p<0.005)$ and $G_{\max }(p<0.033)$ between the control and AahITtreated oocytes. Values for Para/TipE $(C)$ were as follows: $V_{1 / 2}=$ $-19 \pm 2.23, k=6.5 \pm 0.3, G_{\max }=19.42 \pm 1.37$; AahIT-treated oocytes, $V_{1 / 2}=22.2 \pm 2.7, k=6.2 \pm 0.2, G_{\max }=26.31 \pm 2.84$; showing significant differences (paired two-tailed $t$ test) in $V_{1 / 2}(p<0.007)$ and $G_{\max }(p<$ 0.019 ) between the control and AahIT-treated oocytes. $D$, Normalized currents $\left(I / I_{\max }\right)$, elicited using the prepulse protocol, plotted as a function of membrane voltage $(V)$ in either control (open triangles; $n=7$ ) or $10 \mu \mathrm{M}$ AahIT treated ( filled triangles; $n=4$ ) in rBIIA-expressing oocytes.
rBIIA

A

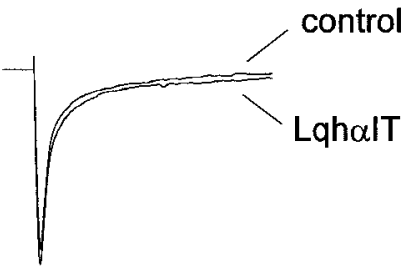

$\frac{1 \mu \mathrm{A}}{5 \mathrm{~ms}}$

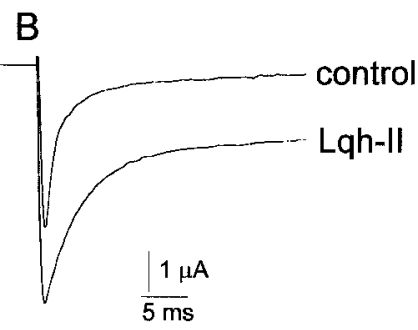

rBIIA-ParaD2
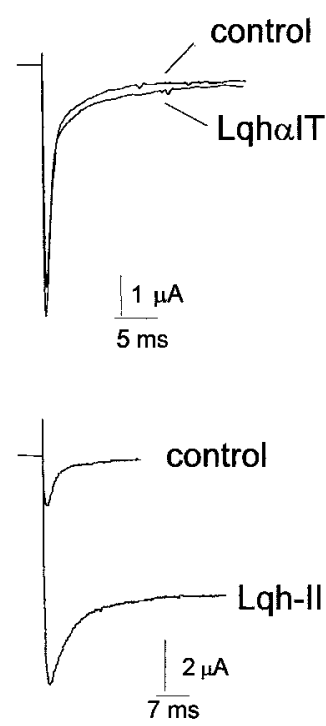

Figure 5. The effect of scorpion $\alpha$-toxins on rBIIA-ParaD2 and rBIIA. Oocytes were held at $-80 \mathrm{mV}$, and maximal rBIIA or rBIIA-ParaD2 currents, recorded every $1 \mathrm{~min}$, were elicited by a depolarizing pulse to -10 or $0 \mathrm{mV}$, respectively. $A, \mathrm{rBIIA}-\mathrm{ParaD} 2$ (right) and rBIIA (left) currents, before (control) and 13 min after application of $1 \mu \mathrm{M} \mathrm{Lqh} \alpha \mathrm{IT}$. Same results were obtained with the toxin at $5 \mu \mathrm{M}$. B, rBIIA-ParaD2 (right) and rBIIA (left) currents, before (control) and 13 min after application of $200 \mathrm{~nm}$ Lqh-II.

Table 1. The effect of scorpion $\alpha$-toxins on the inactivation of sodium current

\begin{tabular}{|c|c|c|c|c|}
\hline & \multicolumn{2}{|l|}{ Lqh-II ${ }^{a}$} & \multicolumn{2}{|l|}{$\operatorname{Lqh} \alpha \mathrm{IT}^{b}$} \\
\hline & $\tau 1(\mathrm{msec})$ & $\tau 2(\mathrm{msec})$ & $\tau 1$ (msec) & $\tau 2(\mathrm{msec})$ \\
\hline \multicolumn{5}{|l|}{ rBIIA } \\
\hline Control & $1.08 \pm 0.1$ & $10.42 \pm 1.3$ & $1.15 \pm 0.1$ & $11.6 \pm 2$ \\
\hline+ Toxin & $3.27 \pm 0.1^{* *}$ & $18.56 \pm 1.4^{*}$ & $1.2 \pm 0.1$ & $11.57 \pm 1.6$ \\
\hline$n$ & \multicolumn{2}{|c|}{5} & \multicolumn{2}{|c|}{6} \\
\hline \multicolumn{5}{|c|}{ rBIIA-ParaD2 } \\
\hline Control & $1.2 \pm 0.2$ & $9.9 \pm 1.4$ & $1.06 \pm 0.1$ & $9.28 \pm 1.1$ \\
\hline+ Toxin & $3.6 \pm 0.2^{* *}$ & $30 \pm 3.1^{*}$ & $1.01 \pm 0.1$ & $7.3 \pm 0.7^{*}$ \\
\hline$n$ & \multicolumn{2}{|c|}{4} & \multicolumn{2}{|c|}{7} \\
\hline
\end{tabular}

Values were measured before and 15 min after application of the indicated toxin. $n$ denotes number of oocytes. Values represent mean \pm SEM, and asterisks represent a significant difference between control and toxin-treated oocytes. ${ }^{*} p \leq 0.05$; $* * p \leq 0.01$.

${ }^{a}$ At least $100 \mathrm{~nm}$ was used to obtain a maximal effect of Lqh-II.

${ }^{b}$ One to $5 \mu \mathrm{M} \mathrm{Lqh} \alpha \mathrm{IT}$ were used.

\section{Domain 2 of the insect channel confers $\mathrm{NaCh}$ sensitivity to AahIT}

Two apparent effects on $\mathrm{Na}^{+}$current activation were documented for the anti-insect toxin AahIT in neuronal preparations of various insects: an increase of maximal current and a negative shift of the voltage dependence of activation (Zlotkin, 1997). Thus, AahI T increased the $\mathrm{Na}^{+}$peak current in cockroach axons and shifted the activation curve (Zlotkin, 1997), whereas, in intact insect neurons (Heliothis virescens, Lepidoptera), only the shift in the activation was observed (Lee and Adams, 2000). In the Drosophila (fruit fly) Para $\mathrm{NaCh}$ coexpressed with TipE in oo- 
cytes, the main effect of AahIT was an increase of the peak current (by $35 \%$ ) accompanied by a small shift $(\sim 3 \mathrm{mV})$ in the activation curve (Fig. 4C). It appears that the sensitivity to AahIT varies among the different insect species and may depend also on channel preparation (native channels in neurons vs expressed channels in oocytes). Notably, AahIT, at concentrations similar to those used for Drosophila Para NaCh, exerted both effects on the rBIIA-ParaD2: an increase in the peak current (by 20\%) and a shift in the activation curve (by $\sim 6 \mathrm{mV}$ ) (Fig. $3 B$ ). In view of the similar toxin sensitivity of the chimeric channel to the insect Para channel, together with the absolute toxin resistance of the mammalian channel rBIIA (Fig. 1), these results indicate that the receptor site for the insect-selective toxin AahIT resides mainly in ParaD2. Also, TipE is apparently not involved in the toxin selectivity to insect channels, because the chimera was sensitive to AahIT in its absence.

Interestingly, the concentrations of AahIT required to elicit the effects in neurons (50-100 nM) (Lee and Adams, 2000) were 10-fold lower than those required for the chimeric and Drosophila Para channels $(1-2 \mu \mathrm{M})$. These differences may be attributed to variations in susceptibility of various insects to the toxin (Fishman et al., 1997), which most probably vary in their NaCh Para gene homologs. Such variations may be exemplified by Sarcophaga blowfly larvae, which are at least 130 -fold more sensitive to AahIT than Drosophila melanogaster wild-type flies. Moreover, in various Drosophila strains, which differ by a few substitutions in their gene encoding the Para channel, the sensitivity to AahIT is reduced by up to 2000 -fold compared with blowflies (Zlotkin et al., 1999).

The negative shift in the voltage sensitivity of activation induced by AahIT on insect and the rBIIA-ParaD2 channels resembles the effect of scorpion $\beta$-toxins on mammalian channels (Cahalan, 1975; Meves et al., 1982; Wang and Strichartz, 1983; Vijverberg et al., 1984; Jonas et al., 1986; Marcotte et al., 1997; Cestele et al., 1998; Tsushima et al., 1999). Furthermore, the effects of AahIT on rBIIA-ParaD2 was potentiated by a depolarizing prepulse (Fig. 4), as was shown previously for some $\beta$-toxins (Cestele et al., 1998; Tsushima et al., 1999), thus establishing the similarity between AahIT and $\beta$-toxins modes of action.

\section{The rBIIA-ParaD2 acquired additional insect NaCh properties}

In addition to the sensitivity to AahIT, the rBIIA-ParaD2 acquired inherent activation parameters [a positive shift of the half activation voltage $\left(V_{1 / 2}\right)$ ] (Fig. $2 D$ ) that were similar to those of the intact Drosophila Para channel expressed in oocytes (Warmke et al., 1997) (Fig. 4C). The critical impact of the origin of D2 on the voltage-dependent activation of $\mathrm{NaChs}$ was demonstrated previously (Marcotte et al., 1997). In view of the $65 \%$ identity between the entire D2 of rBIIA and Drosophila Para, sharing, however, a $100 \%$ identity in their voltage sensors (D2-S4), our results highlight the importance of structural elements other than $\mathrm{D} 2-\mathrm{S} 4$ in the activation process of $\mathrm{NaChs}$. Indeed, in addition to the previously demonstrated critical impact of mutations in D2-S4 (Stuhmer et al., 1989; Auld et al., 1990; Moran et al., 1994), changes in the external loops of D2 in mammalian NaChs were also shown to affect the voltage dependence of activation (Cestele et al., 1998; Qu et al., 1999).

Other insect properties conferred by the insect D2 were revealed by analyzing the effect of the site-3 $\alpha$-toxin Lqh-II on the chimeric channel. Lqh-II affected the inactivation of rBIIAParaD2 more potently than that of rBIIA (Fig. 5B, Table 1). This effect was not accompanied by a change in the toxin binding affinity (Fig. 5), supporting the notion that D2 does not contribute to receptor site-3 (Gordon et al., 2002). Because D2 has not yet been implicated in any phenomenon pertaining to $\mathrm{NaCh}$ inactivation, our results indicate involvement of D2 in inactivation, in addition to its expected involvement in activation (as detailed above).

\section{Additional considerations}

Two additional inferences regarding $\mathrm{NaChs}$ can be made on the basis of the results obtained in this study. (1) The findings that the binding of both $\alpha$-toxins Lqh-II and Lqh $\alpha$ IT were not affected by the change in D2 (Fig. 5) indicate that the selectivity of $\alpha$-toxins toward rat brain and insect $\mathrm{NaCh}$ is not conferred by $\mathrm{D} 2$. (2) The insect Para Drosophila $\mathrm{NaCh}$ is hardly expressed in oocytes without the coexpression of the auxiliary subunit TipE (Feng et al., 1995; Warmke et al., 1997). We show, however, that the mammalian channel rBIIA can acquire insect channel properties by virtue of insertion of a single domain of Para Drosophila channel without the need of TipE. Thus, the chimera approach presents a technological advantage for the study of $\mathrm{NaChs}$ in heterologous systems, which circumvents possible difficulties resulting from missing additional subunits.

\section{REFERENCES}

Auld VJ, Goldin AL, Krafte DS, Marshall J, Dunn J, Catterall WA, Lester HA, Davidson N, Dunn RJ (1988) A rat brain $\mathrm{Na}^{+}$channel $\alpha$ subunit with novel gating properties. Neuron 1:449-461.

Auld VJ, Goldin AL, Krafte DS, Catterall WA, Lester HA, Davidson N, Dunn RJ (1990) A neutral amino acid change in segment IIS4 dramatically alters the gating properties of the voltage-dependent sodium channel. Proc Natl Acad Sci USA 87:323-327.

Cahalan MD (1975) Modification of sodium channel gating in frog myelinated nerve fibres by Centruroides sculpturatus scorpion venom. J Physiol (Lond) 244:511-534.

Catterall WA (1992) Cellular and molecular biology of voltage-gated sodium channels. Physiol Rev 72:S15-S48.

Catterall WA (1995) Structure and function of voltage-gated ion channels. Annu Rev Biochem 64:493-531.

Cestele S, Qu Y, Rogers JC, Rochat H, Scheuer T, Catterall WA (1998) Voltage sensor-trapping: enhanced activation of sodium channels by beta-scorpion toxin bound to the S3-S4 loop in domain II. Neuron 21:919-931.

Chen H, Gordon D, Heinemann SH (2000) Modulation of cloned skeletal muscle sodium channels by the scorpion toxins Lqh II, Lqh III, and Lqh alphaIT. Pflügers Arch 439:423-432.

Dascal N, Lotan I (1991) Activation of protein kinase C alters voltage dependence of a Na${ }^{+}$channel. Neuron 6:165-175.

Dascal N, Lotan I (1992) Expression of exogenous ion channels and neurotransmitter receptor in RNA-injected Xenopus oocytes. In: Methods in molecular biology (protocols in molecular neurobiology) (Longstaff A, Revest P, eds), pp 205-225. Totowa, NJ: Humana.

De Lima ME, Martin MF, Diniz CR, Rochat H (1986) Tityus serrulatus toxin VII bears pharmacological properties of both beta-toxin and insect toxin from scorpion venoms. Biochem Biophys Res Commun 139:296-302.

Eitan M, Fowler E, Herrmann R, Duval A, Pelhate M, Zlotkin E (1990) A scorpion venom neurotoxin paralytic to insects that affects sodium current inactivation: purification, primary structure, and mode of action. Biochemistry 29:5941-5947.

Feng G, Deak P, Chopra M, Hall LM (1995) Cloning and functional analysis of TipE, a novel membrane protein that enhances Drosophila para sodium channel function. Cell 82:1001-1011.

Fishman L, Herrmann R, Gordon D, Zlotkin E (1997) Insect tolerance to a neurotoxic polypeptide: pharmacokinetic and pharmacodynamic aspects. J Exp Biol 200:1115-1123.

Gershon E, Weigl L, Lotan I, Schreibmayer W, Dascal N (1992) Protein kinase A reduces voltage-dependent $\mathrm{Na}^{+}$current in Xenopus oocytes. J Neurosci 12:3743-3752.

Gilles N, Blanchet C, Shichor I, Zaninetti M, Lotan I, Bertrand D, Gordon D (1999) A scorpion $\alpha$-like toxin that is active on insects and mammals reveals an unexpected specificity and distribution of sodium channel subtypes in rat brain neurons. J Neurosci 19:8730-8739.

Gilles N, Chen H, Wilson H, Le Gall F, Montoya G, Molgo J, Schonherr R, Nicholson G, Heinemann SH, Gordon D (2000) Scorpion alpha 
and alpha-like toxins differentially interact with sodium channels in mammalian CNS and periphery. Eur J Neurosci 12:2823-2832.

Gilles N, Leipold E, Chen H, Heinemann SH, Gordon D (2001) Effect of depolarization on binding kinetics of scorpion $\alpha$-toxin highlights conformational changes of rat brain sodium channels. Biochemistry 40:14576-14584.

Gordon D (1997a) A new approach to insect-pest control: combination of neurotoxins interacting with voltage sensitive sodium channels to increase selectivity and specificity. Invert Neurosci 3:103-116.

Gordon D (1997b) Sodium channels as targets for neurotoxins: mode of action and interaction of neurotoxins with receptor sites on sodium channels. In: Toxins and signal transduction (Cellular and molecular mechanisms of toxin action) (Gutman Y, Lazarowici P, eds), pp 119149. Amsterdam: Harwood.

Gordon D, Zlotkin E (1993) Binding of an alpha scorpion toxin to insect sodium channels is not dependent on membrane potential. FEBS Lett 315:125-128.

Gordon D, Jover E, Couraud F, Zlotkin E (1984) The binding of the insect selective neurotoxin (AaIT) from scorpion venom to locust synaptosomal membranes. Biochim Biophys Acta 778:349-358.

Gordon D, Moskowitz H, Eitan M, Warner C, Catterall WA, Zlotkin E (1992) Localization of receptor sites for insect-selective toxins on sodium channels by site-directed antibodies. Biochemistry 31:7622-7628.

Gordon D, Martin-Eauclaire MF, Cestele S, Kopeyan C, Carlier E, Benkhalifa R, Pelhate M, Rochat H (1996) Scorpion toxins affecting sodium current inactivation bind to distinct homologous receptor sites on rat brain and insect sodium channels. J Biol Chem 271:8034-8045.

Gordon D, Savarin P, Gurevitz M, Zinn-Justin S (1998) Functional anatomy of scorpion toxins affecting sodium channels. J Toxicol Toxin Rev 17:131-159.

Gordon D, Gilles N, Bertrand D, Molgo J, Nicholson GM, Sauviat MP, Benoit E, Shichor I, Lotan I, Gurevitz M, Kallen RG, Heinemann SH (2002) Scorpion toxins differentiating among neuronal sodium channel subtypes: nature's guide for design of selective drugs. In: Perspectives in molecular toxinology (Menez A, ed). Chichester, UK: Wiley, in press.

Isom LL, De Jongh KS, Catterall WA (1994) Auxiliary subunits of voltage-gated ion channels. Neuron 12:1183-1194.

Jaimovich E, Ildefonse M, Barhanin J, Rougier O, Lazdunski M (1982) Centruroides toxin, a selective blocker of surface $\mathrm{Na}^{+}$channels in skeletal muscle: voltage-clamp analysis and biochemical characterization of the receptor. Proc Natl Acad Sci USA 79:3896-3900.

Jing J, Chikvashvili D, Singer-Lahat D, Thornhill WB, Reuveny E, Lotan I (1999) Fast inactivation of a brain $\mathrm{K}^{+}$channel composed of Kv1.1 and $\mathrm{Kv}$ beta1.1 subunits modulated by $\mathrm{G}$ protein beta gamma subunits. EMBO J 18:1245-1256.

Jonas P, Vogel W, Arantes EC, Giglio JR (1986) Toxin gamma of the scorpion Tityus serrulatus modifies both activation and inactivation of sodium permeability of nerve membrane. Pflügers Arch 407:92-99.

Jover E, Couraud F, Rochat H (1980) Two types of scorpion neurotoxins characterized by their binding to two separate receptor sites on rat brain synaptosomes. Biochem Biophys Res Commun 95:1607-1614.

Kallen RG, Cohen SA, Barchi RL (1993) Structure, function and expression of voltage-dependent sodium channels. Mol Neurobiol 7:383-428.

Kontis KJ, Goldin AL (1993) Site-directed mutagenesis of the putative pore region of the rat IIA sodium channel. Mol Pharmacol 43:635-644.

Lazdunski M, Frelin C, Barhanin J, Lombet A, Meiri H, Pauron D, Romey G, Schmid A, Schweitz H, Vigne P, Vijverberg H (1986) Polypeptide toxins as tools to study voltage-sensitive $\mathrm{Na}^{+}$channels. Ann NY Acad Sci 479:204-220.

Lee D, Adams ME (2000) Sodium channels in central neurons of the tobacco budworm, Heliothis virescens: basic properties and modification by scorpion toxins. J Insect Physiol 46:499-508.

Loughney K, Kreber R, Ganetzky B (1989) Molecular analysis of the para locus, a sodium channel gene in Drosophila. Cell 58:1143-1154.

Ma Z, Tang L, Lu S, Kong J, Gordon D, Kallen RG (2000) The domain 4 s3-s4 extracellular loop provides molecular determinants for binding of $\alpha$-scorpion toxins (Lqh-II, Lqh-III and Lqh $\alpha$ IT) to the voltage-gated rat skeletal muscle $\mathrm{Na}^{+}$channel (rSKM1). Biophys J 78:A1011.

Makita N, Bennett PB, George Jr AL (1996) Molecular determinants of beta 1 subunit-induced gating modulation in voltage-dependent $\mathrm{Na}^{+}$ channels. J Neurosci 16:7117-7127.

Marcotte P, Chen LQ, Kallen RG, Chahine M (1997) Effects of Tityus serrulatus scorpion toxin gamma on voltage-gated $\mathrm{Na}^{+}$channels. Circ Res 80:363-369.

Meves H, Rubly N, Watt DD (1982) Effect of toxins isolated from the venom of the scorpion Centruroides sculpturatus on the $\mathrm{Na}$ currents of the node of Ranvier. Pflügers Arch 393:56-62.

Moran O, Gheri A, Zegarra-Moran O, Imoto K, Conti F (1994) Proline mutations on the S4 segment of rat brain sodium channel II. Biochem Biophys Res Commun 202:1438-1444.

Noda M, Ikeda T, Suzuki H, Takeshima H, Takahashi T, Kuno M, Numa $S$ (1986) Expression of functional sodium channels from cloned cDNA. Nature 322:826-828.

Patton DE, West JW, Catterall WA, Goldin AL (1992) Amino acid residues required for fast $\mathrm{Na}^{+}$-channel inactivation: charge neutralizations and deletions in the III-IV linker. Proc Natl Acad Sci USA 89:10905-10909.

Pelhate M, Zlotkin E (1981) Voltage dependent slowing of the turn off $\mathrm{Na}+$ current in the cockroach giant axon induced by the scorpion venom "insect toxin." J Physiol (Lond) 319:30-31.

Pelhate M, Zlotkin E (1982) Actions of insect toxin and other toxins derived from the venom of the scorpion Androctonus australis in isolated giant axons of the cockroach (Periplaneta americana). J Exp Biol 97:67-77.

Qu Y, Rogers JC, Chen SF, McCormick KA, Scheuer T, Catterall WA (1999) Functional roles of the extracellular segments of the sodium channel alpha subunit in voltage-dependent gating and modulation by beta1 subunits. J Biol Chem 274:32647-32654.

Rogers JC, Qu Y, Tanada TN, Scheuer T, Catterall WA (1996) Molecular determinants of high affinity binding of alpha-scorpion toxin and sea anemone toxin in the S3-S4 extracellular loop in domain IV of the $\mathrm{Na}^{+}$channel alpha subunit. J Biol Chem 271:15950-15962.

Sautiere P, Cestele S, Kopeyan C, Martinage A, Drobecq H, Doljansky Y, Gordon D (1998) New toxins acting on sodium channels from the scorpion Leiurus quinquestriatus hebraeus suggest a clue to mammalian vs insect selectivity. Toxicon 36:1141-1154.

Stuhmer W, Conti F, Suzuki H, Wang XD, Noda M, Yahagi N, Kubo H, Numa S (1989) Structural parts involved in activation and inactivation of the sodium channel. Nature 339:579-603.

Thomsen WJ, Catterall WA (1989) Localization of the receptor site for alpha-scorpion toxins by antibody mapping: implications for sodium channel topology. Proc Natl Acad Sci USA 86:10161-10165.

Tsushima RG, Borges A, Backx PH (1999) Inactivated state dependence of sodium channel modulation by beta-scorpion toxin. Pflügers Arch 437:661-668

Vais H, Williamsom MS, Goodson SJ, Devonshire AL, Warmke JW, Usherwood PNR, Cohen CJ (2000) Activation of Drosophila sodium channels promotes modification by Deltamethrin; reductions in affinity caused by knock-down resistance mutations. J Gen Physio 115:305-318

Vijverberg HP, Pauron D, Lazdunski M (1984) The effect of Tityus serrulatus scorpion toxin gamma on $\mathrm{Na}$ channels in neuroblastoma cells. Pflügers Arch 401:297-303.

Wallner M, Weigl L, Meera P, Lotan I (1993) Modulation of the skeletal muscle sodium channel alpha-subunit by the beta 1-subunit. FEBS Lett 336:535-539.

Wang GK, Strichartz GR (1983) Purification and physiological characterization of neurotoxins from venoms of the scorpions Centruroides sculpturatus and Leiurus quinquestriatus. Mol Pharmacol 23:519-533.

Warmke JW, Reenan RA, Wang P, Qian S, Arena JP, Wang J, Wunderler D, Liu K, Kaczorowski GJ, Van der Ploeg LH, Ganetzky B, Cohen CJ (1997) Functional expression of Drosophila para sodium channels. Modulation by the membrane protein TipE and toxin pharmacology. J Gen Physiol 110:119-133.

Zlotkin E (1997) Insect selective neurotoxins from scorpion venoms affecting sodium conductance. In: Toxins and signal transduction (cellular and molecular mechanisms of toxin action) (Lazarowici P, Gutman Y, eds), pp 95-117. Amsterdam: Harwood.

Zlotkin E (1999) The insect voltage-gated sodium channel as target of insecticides. Annu Rev Entomol 44:429-455.

Zlotkin E, Rochat H, Kopeyan, Miranda F, Lissitzky S (1971) Purification and properties of the insect toxin from the venom of the scorpion Androctonus australis Hector. Biochimie 53:1073-1078.

Zlotkin E, Miranda F, Rochat H (1978) Chemistry and pharmacology of Buthinae scorpion venoms. In: Arthropods venoms (Bettini S, ed), pp 317-369. New York: Springer.

Zlotkin E, Kadouri D, Gordon D, Pelhate M, Martin MF, Rochat H (1985) An excitatory and a depressant insect toxin from scorpion venom both affect sodium conductance and possess a common binding site. Arch Biochem Biophys 240:877-887.

Zlotkin E, Devonshire AL, Warmke JW (1999) The pharmacological flexibility of the insect voltage gated sodium channel: toxicity of AaIT to knockdown resistant (kdr) flies. Insect Biochem Mol Biol 29:849853. 